\title{
Dicarbon molecule in the interstellar clouds $\dagger$
}

\author{
Maja Kaźmierczak ${ }^{1}$, Mirosław Schmidt ${ }^{2}$, and Jacek Krełowski ${ }^{1}$ \\ ${ }^{1}$ Center for Astronomy of Nicolaus Copernicus University, \\ ul. Gagarina 11, 87-100 Toruń, Poland \\ email: maja.kazmierczak@gmail.com \\ ${ }^{2}$ Nicolaus Copernicus Astronomical Center, ul. Rabiańska 8, 87-100 Toruń, Poland
}

\begin{abstract}
We present high-resolution and high signal-to-noise spectroscopic observations of interstellar molecular lines of $\mathrm{C}_{2}$ towards early-type stars. $\mathrm{C}_{2}$ is particularly interesting because it is the simplest multicarbon molecule and its abundances give information on the chemistry of interstellar clouds, especially on the pathway of formation of (hydro)carbon chains and PAHs which may be considered as possible carriers of diffuse interstellar bands (DIBs). Homonuclear diatomic molecules have negligible dipol moments and hence radiative cooling of excited rotational levels may go only trough the slow quadrupole transitions (van Dishoeck \& Black 1982). In $\mathrm{C}_{2}$, pumped by galactic average interstellar field rotational levels are excited effectively much above the gas kinetic temperature and a rotational ladder of electronic transitions is usually observed from high rotational levels. Relations between abundances of the dicarbon and other simple interstellar molecules are considered as well.
\end{abstract}

Keywords. ISM: molecules, ISM: abundances

\section{Introduction}

The observational material consists of a set of high-quality echelle spectra (with resolution of 110,000 and signal-to-noise ratio above 200) from the high-resolution spectrograph UVES, acquired at the ESO Paranal Observatory (Bagnulo et al. 2003). Dicarbon is easily available observationally through its electron-vibration-rotation transitions from ground electronic level $X^{1} \Sigma_{g}^{+}$to excited electronic levels $A^{1} \Pi_{u}$ (Phillips band) in optical and

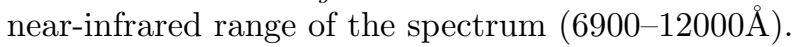

\section{Results}

We measured equivalent widths of (1-0) 10133-10262, , (2-0) 8750-8849 $\AA$, (3-0) 7714$7793 \AA$ and in one case (HD 147889) also (4-0) 6909-6974 $\AA$ bands of the $\mathrm{C}_{2}$ Phillips system. The list of observed objects with some basic parameters is presented in Table 1. There are also determined total column densities of $\mathrm{C}_{2}$ toward each star and excitation temperatures, which were extracted from the fit to the first four rotational levels $\left(J^{\prime \prime}=\right.$ $0,2,4,6)$.

In cases of optically thin clouds, i. e. for weak lines the column densities are derived from the relationship: $N_{\text {col }}\left(J^{\prime \prime}\right)=1.13 * 10^{17} \mathrm{EW} /\left(f_{i j} * \lambda\right)$, where $\lambda$ is the wavelength in $[\AA]$, $f_{i j}$ is the absorption oscillator strength and EW equivalent widths in $[\mathrm{m} \AA]$. Total column density is a sum of column densities determined from each low rotational level $N_{\text {col }}\left(J^{\prime \prime}\right)$. Error of column densities depends on EW, which follows quality of spectrum: resolution and signal-to-noise ratio. Column density depends also on the oscillator strengths. We compare their theoretical values with our observations (to check their correctness), building relations between equivalent widths for transitions originated in each low rotational level, according to equation: $E W 1 / E W 2=f 1 / f 2$. Observationally determined ratios of oscillator strengths are very similar to theoretical ones (from Bakker et al. 1997). Some

$\dagger$ Based on observations collected at the European Southern Observatory, Paranal, Chile 
Table 1. Basic parameters of program stars and results for $C_{2}$ toward these objects

\begin{tabular}{|c|c|c|c|c|}
\hline object & $T_{\operatorname{exc}}[K]$ & $N_{\mathrm{col}}\left[10^{13} / \mathrm{cm}^{2}\right]$ & $\mathrm{Sp} / \mathrm{L}$ & $\mathrm{E}(\mathrm{B}-\mathrm{V})$ \\
\hline HD 76341 & $66 \pm 16$ & $1.20 \pm 0.2$ & O $9 \mathrm{Ib}$ & 0.49 \\
\hline HD 96917 & $66 \pm 16$ & $1.36 \pm 0.4$ & $\mathrm{O} 8.5 \mathrm{Ib}(\mathrm{f})$ & 0.32 \\
\hline HD 97253 & $73 \pm 68$ & $1.27 \pm 0.3$ & O 5.5 IIIf,cl, el & 0.45 \\
\hline HD 115363 & $60 \pm 1$ & $4.01 \pm 0.5$ & B $1 \mathrm{Ia}$ & 0.69 \\
\hline HD 136239 & $56 \pm 11$ & $3.66 \pm 0.9$ & $\mathrm{~B} 1.5 \mathrm{Ia} \mathrm{v}$ & 0.94 \\
\hline HD 147933 & $63 \pm 35$ & $5.86 \pm 0.3$ & B $2.5 \mathrm{~V}$ & 0.45 \\
\hline HD 147889 & $73 \pm 11$ & $12.6 \pm 0.8$ & B $2 \mathrm{~V}$ & 1.07 \\
\hline HD 148184 & $67 \pm 18$ & $2.8 \pm 0.3$ & B $2 \mathrm{Vn}, \mathrm{el}$ & 0.52 \\
\hline HD 148379 & $81 \pm 22$ & $1.47 \pm 0.2$ & B $1.5 \mathrm{Ia} \mathrm{el}$ & 0.61 \\
\hline HD 151932 & $87 \pm 12$ & $1.57 \pm 0.2$ & WN $7 \mathrm{~A}$ wr & 0.35 \\
\hline HD 152003 & $76 \pm 2$ & $2.62 \pm 0.6$ & $09.7 \mathrm{Iab} \mathrm{v}$ & 0.53 \\
\hline HD 152270 & $67 \pm 27$ & $2.56 \pm 0.8$ & W C $7 \mathrm{wr}$ & 0.53 \\
\hline HD 154368 & $53 \pm 6$ & $5.6 \pm 0.7$ & $09.5 \mathrm{Iab}$ & 0.78 \\
\hline HD 161056 & $80 \pm 18$ & $3.18 \pm 0.5$ & B $1.5 \mathrm{~V}$ & 0.60 \\
\hline HD 163800 & $59 \pm 22$ & $2.60 \pm 0.6$ & O 7IIIf & 0.60 \\
\hline HD 168607 & $89 \pm 10$ & $1.35 \pm 0.2$ & B 9Iape & 1.61 \\
\hline HD 168625 & $70 \pm 24$ & $1.32 \pm 0.5$ & B 6 I ap & 1.49 \\
\hline HD 169454 & $39 \pm 3$ & $5.80 \pm 0.6$ & B 1 Iael & 0.93 \\
\hline HD 179406 & $48 \pm 9$ & $4.60 \pm 0.6$ & B $3 \mathrm{~V}$ & 0.33 \\
\hline BD-14 5037a & $53 \pm 5$ & $6.38 \pm 0.6$ & B $1.5 \mathrm{Ia}$ & 1.59 \\
\hline $\mathrm{BD}-145037 \mathrm{~b}$ & $64 \pm 4$ & $4.26 \pm 0.6$ & & \\
\hline
\end{tabular}
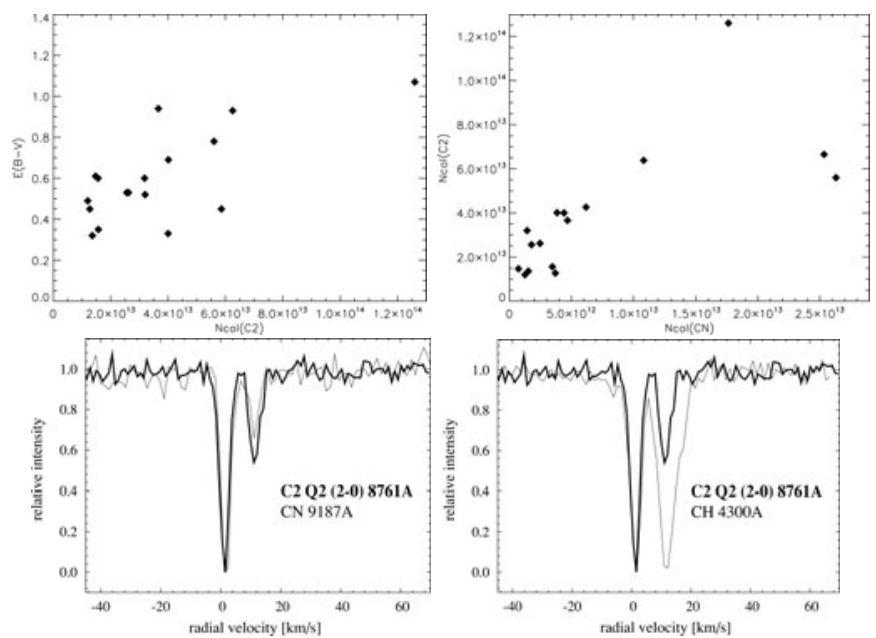

Figure 1. (a) [Top left] $N_{\text {col }}\left(\mathrm{C}_{2}\right)-\mathrm{E}(\mathrm{B}-\mathrm{V})(\mathrm{b})$ [Top right] $N_{\mathrm{col}}(\mathrm{CN})-N_{\mathrm{col}}\left(\mathrm{C}_{2}\right)$ (c) [Bottom left] $\mathrm{C}_{2}-\mathrm{CN}(\mathrm{d})$ [Bottom right] $\mathrm{C}_{2}-\mathrm{CH}$

departures likely follow errors in measuring $\mathrm{C}_{2}$ lines. For the strongest lines, especially from (2-0) vibrational band, the relations evidently confirm theoretical expectations.

We measured dicarbon column densities toward above 20 stars. After that we tried to correlate $\mathrm{C}_{2}$ abundance with those of other interstellar molecules $(\mathrm{CN}$ and $\mathrm{CH}$ ) and with extinction (Figure 1a). Such relations may constrain chemical pathways which lead to the formation of these species.

Figure $1 \mathrm{~b}$ shows relation between column densities of $\mathrm{C}_{2}$ and $\mathrm{CN}$. A majority of the considered objects suggest a tight correlation, but two points are considerably discrepant. These represent HD 169454 and HD 154368. Physico-chemical conditions in the intervening interstellar clouds (e.g., very low $T_{\text {exc }}$ ) likely vary considerably in these objects while compared to average conditions. Figures 1c and 1d compare Doppler-splitted profiles of molecular features in the spectrum of BD-14 5037. We compare lines profiles of $\mathrm{C}_{2}(\mathrm{Q}(2)$ $8761 \AA$ of (2-0) band) and $\mathrm{CN}(9187 \AA)$ which suggests that $\mathrm{C}_{2}$ is spatially correlated with CN. Figure 1d compares $\mathrm{C}_{2}$ and $\mathrm{CH}(4300 \AA)$ in the spectrum of BD-14 5037. CH does not share the profile shape with $\mathrm{C}_{2}$.

\section{References}

Bagnulo, S., Jehin, E., Ledoux, C., Cabanac, R., Melo, C., Gilmozzi, R., \& The ESO Paranal Science Operations Team 2003, The Messenger, 114, 10

Bakker, E. J., van Dishoeck, E. F., Waters, L. B. F. M., \& Schoenmaker, T. 1997, A $\&$ A, 323, 469 van Dishoeck, E. F., \& Black, J. 1982, ApJ, 258, 533 